\title{
Design of Lidar System Based on Marine Oil Spill Monitoring
}

\author{
LI Jing ${ }^{1,2 *}$, CHE Ying ${ }^{1}$, Luan Shuang ${ }^{2}$, Wang Zhaoxin ${ }^{2}$, Yang Kun ${ }^{2}$ \\ ${ }^{1}$ College of Optoelectronic Engineering, Changchun University of Science and Technology, Changchun, Jilin 130022, \\ China \\ ${ }^{2}$ Aviation University of Air Force, Changchun, Jilin 130022, China
}

\begin{abstract}
The occurrence of offshore oil spill will cause great harm to Marine natural ecology and Marine living resources, and seriously affect Marine aquaculture, fishery, tourism and other Marine industries. In the field of monitoring oil spill accident, traditional passive remote sensing measurement is still relying on, and the monitoring of oil spill accident occurring at night is not very good. According to the condition of the surface of the oil spill at night monitoring requirements, design and development of laser radar for monitoring the sea are permeated with oil pollution. This article mainly from the super continuous spectrum of light sources and monochrome laser radar detector selection, spectrometer wavelength calibration, the structure of the optical system design, radar receiving system image quality evaluation, the mechanical structure design and mechanics analysis and prototype design aspects, introduces the first system design of laser radar in detail.The design indexes of lidar system for monitoring oil spill pollution meet the technical requirements.
\end{abstract}

\section{Introduction}

The occurrence of offshore oil spill will cause great harm to the natural ecology of the ocean and Marine living resources, and seriously affect Marine aquaculture, fishery, tourism and other Marine industries. In this paper, starting from the monitoring of oil pollution in the sea, according to the specific requirements of technical indicators for monitoring oil pollution in the sea, the design, manufacture, adjustment and other key technologies of the new full-time multispectral lidar were studied in depth.

\subsection{Research Background}

In 1989, an American oil tanker, exxon valdez, ran aground and spilled more than 30,000 tons of oil, killing about 4,000 sea otters and 100,000 seabirds. In 2011, the penglai oil field in bohai sea caused the submarine bottom fault zone to break up suddenly under the pressure of oil production during the process of oil production, resulting in the occurrence of well kick. The accident polluted the sea water of about 5,500 square kilometers, equivalent to $7 \%$ of the area of the bohai sea. In 2018, the changfeng crystal collided with the Panamanian oil tanker sanchi about 160 nautical miles east of the Yangtze estuary. Sanchi, carrying 136,000 tons of condensate oil and 1,000 tons of heavy diesel oil, burned and eventually exploded. The spillovers spread to the sea of Japan along with the opposite mare current, causing great harm to the ecological environment and the safety of people's property ${ }^{[1-2]}$. Therefore, it is necessary to monitor the occurrence of Marine oil spill in a timely and accurate manner.

\subsection{Research Significance}

As the oil spill is an emergency, the time and place of its occurrence are random, so it is necessary to monitor its occurrence 24 hours a day. The commonly used methods for monitoring oil spills are mainly remote sensing, including near-infrared remote sensing technology, ultraviolet remote sensing technology, synthetic aperture radar, hyperspectral, laser fluorescence method ${ }^{[3-6]}$, etc. The monitoring methods currently used have their own disadvantages. For example, synthetic aperture radar USES microwave radiometric remote sensing technology, which has the advantage of having a large detection range and being able to operate continuously 24 hours a day. However, it has a low resolution and cannot accurately judge the oil film thickness ${ }^{[7]}$. Nearinfrared remote sensing technology has the advantage of high reliability and high speed, but it is difficult to distinguish the thickness of oil film ${ }^{[8]}$. Ultraviolet band also has a high radiation degree for extremely thin oil layer, but the ultraviolet band has a fast attenuation in the atmosphere and cannot be used for remote distance [9].By emitting ultraviolet light of specific bands, the laser fluorescence method can stimulate oil spill and produce fluorescence, thus realizing 24-hour uninterrupted detection of oil spill. However, the fluorescence intensity is weak and susceptible to interference, so its accuracy is not high. In view of the above problems, this paper starts from the monitoring of

\footnotetext{
*LiJing: 568026388@qq.com
} 
offshore oil pollution, and according to the specific requirements of the technical indicators of offshore oil pollution monitoring, carries out in-depth research on the design, manufacture, adjustment and calibration of the new full-time multispectral stimulated optical radar and other key technologies.

\section{2 lidar initial system selection}

The multi-spectral lidar optical system requires the working band to cover $400 \mathrm{~nm}-1100 \mathrm{~nm}$, the laser transmission power is greater than $1 \mathrm{~mW} / \mathrm{nm}$, the beam spreading ratio is greater than 10 , the bandwidth is better than $0.5 \mathrm{~nm}$, and the wavelength accuracy in the whole spectrum regulation range is better than $0.2 \mathrm{~nm}$. The multispectral lidar also needs to have the ability of visible light and sight. The field of view of the visible lidar is greater than $1.6^{\circ}$, and the angular resolution is better than $0.012 \mathrm{mrad}$. To ensure the detection capability of the receiving system, the receiving system $\mathrm{F} \#$ is less than 10. Based on the above factors, the size of commercially available detectors and the design experience of optical system, the optical indexes of lidar are shown in table 1.

Table 1. lidar parameters

\begin{tabular}{cc}
\hline parameter & The numerical \\
\hline Wavelength $(\mathrm{nm})$ & $400-1100$. \\
Telescopic system F\# & 3 \\
Field of view $\left({ }^{\circ}\right)$ & $2.06^{\circ} \times 1.55^{\circ}$ \\
Spatial resolution $(\mathrm{mrad})$ & 0.0128 \\
Laser beam expansion ratio & 8 \\
Operating distance $(\mathrm{km})$ & $>8$ \\
\hline
\end{tabular}

In general, the lidar transmitting system and the receiving system are separated, each with an optical system. The US AN/ FPQ-6 radar uses this technology for ground-based measurement and tracking satellites, the Galileo telescope for laser beam expansion, and the fold-reflection configuration for the echo receiving system. The $20 \mathrm{~cm}$ aperture optical system can realize the tracking measurement of $1000 \mathrm{~km}$ target. To realize miniaturization and intensive, system design scheme uses reflection of light path technology, realizing the laser beam expander and receive one design.Under the condition of sharing a telescopic system, satisfy excitation light beam expander with laser receiving and also can meet the needs of visible light laser radar tracking target, realizing intensive optical system overall design. The reversion system can greatly reduce the number of optical components in the optical system. The system design scheme uses the complex structure of Cassegrain $\mathrm{r}-\mathrm{c}$ and the co-optimization design of multiple structures to complete the common optical path structure of the transmitting system and the receiving system. In order to avoid the energy loss and beam shielding caused by the center block, the laser emitting system adopts the partial circumferential application.

\subsection{Supercontinuum laser white light source}

Supercontinuum white light laser light source is proposed to use sc-pro excited light source of YSL Photonics company. The emission spectrum of the light source covers $400 \mathrm{~nm}-2400 \mathrm{~nm}$, and the spectral energy distribution is shown in figure 1. Energy stability is \pm $1 \%$; time jitter after preheating is $<2 \mathrm{~s}$; pulse energy is $>1 \mathrm{~J}$, including visible power is $>1200 \mathrm{mw}$. The spatial mode of the emitting laser is single-mode Gaussian TEM00 mode, non-polarized. The laser light source is compact,Small size and light source picture is shown in figure2.Mechanical/environmental/electronic specifications are shown in table 2 and the light source can be free of maintenance.

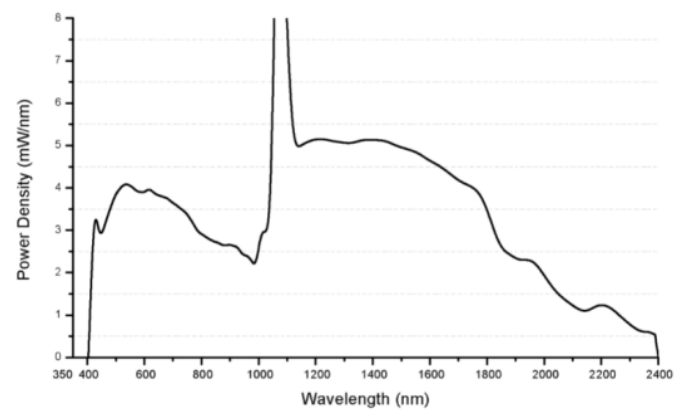

Fig. 1. spectral energy distribution of SC PRO light source $(4 \mathrm{MHz})$

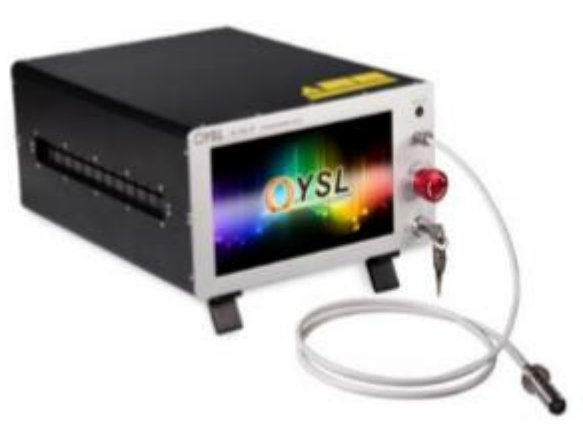

Fig. 2. photo of SC PRO light source

Table 2. sm-30-400 mechanical/environmental/electronic specifications

\begin{tabular}{cc}
\hline category & indicators \\
\hline Control interface and interface & Front panel with USB excuse \\
Working temperature & $+5{ }^{\circ} \mathrm{C}$ to $+45^{\circ} \mathrm{C}$ \\
Weight & $<3 \mathrm{~kg}$ \\
Size $\left(\mathrm{L}^{*} \mathrm{~W} * \mathrm{H}\right)$ & $185 * 85 * 90 \mathrm{~mm}$ \\
Power demand & $100-240-\mathrm{v}, 50 / 60 \mathrm{hz}$ \\
\hline
\end{tabular}

\section{2 detector Selection}

EEV EV76C570 chip is proposed for the detector, the frame frequency of the detector can reach $60 \mathrm{fps}$, the typical quantum efficiency is $47 \%$, the dark noise is $22 \mathrm{e}$-, the pixel number is $1602 \times 1202$, and the interface after the development of the detector is GigE. The complete 
structure of the optical receiving system is shown in figure 3 .

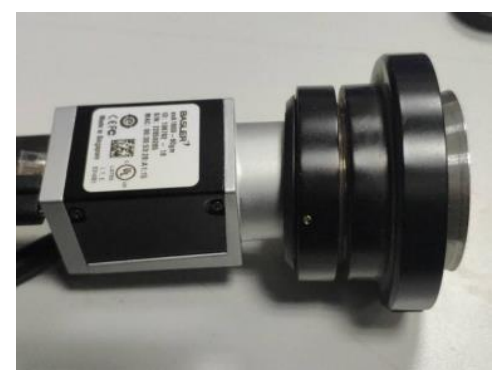

Fig. 3. detector and lens structure

\subsection{Selection of laser beam expander}

The laser beam reamer is intended to be a sigma lbe-10 multiband beam reamer, and the optical system is an air gap lens without gluing lenses, so high-energy lasers can also be used. The optical system adopts Galileo lens structure, which reduces the number of lenses for correcting aberrations and shortens the whole length of the beam expander. Visible light type of beam expander, can be installed directly in the laser outlet. Specific indicators are shown in table 3.

Table 3. index of laser beam expande

\begin{tabular}{cc}
\hline category & indicators \\
\hline Design wavelength & $400 \sim 700 \mathrm{~nm}$ \\
Beam expander ratio & Ten times \\
Cylinder length A & The $127.9 \mathrm{~mm}$ \\
B & The $121.9 \mathrm{~mm}$ \\
Effective incident diameter & Phi is $1.7 \mathrm{~mm}$ \\
The laser damage threshold is $※$ & $4 \mathrm{j} / \mathrm{cm} 2$ \\
The quality of & $0.18 \mathrm{~kg}$ \\
The framework material & Aluminum alloy \\
Frame finish & Black oxidation \\
\hline
\end{tabular}

\subsection{Spectrometer selection}

The spectrometer is intended to use the USB4000 optical fiber spectrometer of ocean optics. As one of the most popular spectrometers in the world, the spectrometer has excellent compatibility and performance. The detector of the spectrometer is Toshiba TCD1304AP linear array CCD, and the detection range reaches $200 \mathrm{~nm}-1100 \mathrm{~nm}$, which can meet the measurement of the visible band. The detector indicators are shown in table 4.Signal-tonoise ratio $300: 1$, dynamic range $2 \times 108$, spectral resolution up to $0.3 \mathrm{~nm}$ (FWHM).

Table 4. spectral indexes of USB4000 spectrometer

\begin{tabular}{cc}
\hline category & indicators \\
\hline Wavelength range & Selection by grating \\
Spectral resolution & 0.3 to $10.0 \mathrm{~nm} \mathrm{FWHM}$ \\
Signalto noise ratio & $300: 1$ (full signal)
\end{tabular}

$\begin{array}{cc}\text { Dark noise } & 16 \\ \text { Dynamic range } & 12 \text { the RMS count } \\ \text { Integration time } & 2 \times 108 \text { (system), 1300:1 single probe } \\ \text { Stray light } & 3.8 \text { milliseconds to } 10 \text { minutes } \\ & \text { At } 600-\mathrm{nm}<0.05 \% ; \\ \text { At } 435 \mathrm{~nm}, 0.10 \%\end{array}$

\section{Optical structure design}

The hyper-continuous spectrum lidar used for oil spill observation is a remote sensing optical instrument. The advantage of reflecting telescopes is that they have no chromatic aberration, so they can detect a wide spectrum. According to the number of mirrors, the total reflection structure can be divided into single lens reflex, two trans, three trans, etc. According to whether the mirror is coaxial with the incident light path, it can be divided into the same axis reflection and off-axis reflection. Among them, the single-lens reflex and two-trans are simple in structure and easy in design, but because of their simple structure and few structure variables, they are not enough to correct the aberration well and achieve satisfactory results. Since the object image is on the same side, it is difficult to choose the appropriate detector and placement position. Adding a mirror can solve this problem, but it will complicate the overall optical design. The off-axis three-trans structure has the advantages of maintaining the coaxial three-trans, as well as the advantages of large field of view, high light transmittance and large flux. However, the off-axis three trans structure has many difficulties, such as complex design, high cost of reflector, and difficult installation and adjustment. To sum up, it is difficult to balance the cost and performance with the fully reflective structure design, so the retractable structure is considered. The reversion structure usually adopts the combination of two reflective systems with optical focal power and a refracting prism without optical focal power. Therefore, it is decided to adopt the retractable structure design as the far-seeing part of the super-continuum fiber lidar.

\subsection{System optical structure design}

In order to simplify the system design and take into account the powerful design capability of software complexity, the design model can be analyzed from the basis of the inverse system. According to the aberration theory of the anti-reflection optical system, there are the following relations:

$$
\begin{aligned}
& S_{I}=\left[\frac{\alpha(\beta-1)^{2}(\beta+1)}{4}-\frac{\alpha(\beta-1)^{3}}{4} e_{2}^{2}\right]-\frac{\beta^{3}}{4}\left(1-e_{1}^{2}\right) \\
& S_{I I}=\frac{1-\alpha}{\alpha}\left[\frac{\alpha(\beta+1)^{3}}{4}-\frac{\alpha(\beta-1)^{2}(\beta+1)}{4 \beta}\right]-\frac{1}{2}
\end{aligned}
$$




$$
\begin{gathered}
S_{I I I}=\left(\frac{1-\alpha}{\alpha}\right)^{2}\left[\frac{\alpha(\beta-1)^{2}(\beta+1)}{4 \beta^{2}}-\frac{\alpha(\beta-1)^{3}}{4 \beta^{2}} e_{2}^{2}\right] \\
-\frac{(1-\alpha)(\beta+1)(\beta-1)}{\alpha \beta}-\frac{\alpha \beta-\beta-1}{\alpha} \\
S_{I V}=\beta-\frac{1+\beta}{\alpha} \\
\left\{\begin{array}{l}
e_{1}^{2}=1+\frac{2 \alpha}{(1-\alpha) \beta^{2}} \\
e_{2}^{2}=\frac{2 \beta}{(1-\alpha)}+(1+\beta)(1-\beta)^{2} \\
(1+\beta)^{3}
\end{array}\right.
\end{gathered}
$$

In this design, $\mathrm{R}-\mathrm{C}$ structure is used as the original structure to fold and reflect the optical system, and the aspheric coefficient is gradually cancelled by increasing the transmission structure. According to the requirements of the optical system, the optical system design of multispectral lidar is realized by using the multi-structure optimization scheme.

Multispectral lidar includes laser emission system, hyperspectral detector and visible light detector. Among them, the hyperspectral detector and the visible light receiving system share the same set of the main body of the telescopic system. The main part of the telescopic system is composed of the primary mirror and the secondary mirror, both of which are folded reflection structures. This main structure is relatively small in chromatic aberration due to the adoption of folded mirror body, and can also be used to correct aberrations at wide wavebands. Between the main part of the far-seeing system of the transmitting system and the back-end lens set is a beam splitter prism, which is mainly used for stripping the hyperspectral detector, and the prism is coated with a semi-inverted and semi-permeable film of $400 \mathrm{~nm}-1100 \mathrm{~nm}$ in the middle.

The transmission part is connected to the visible light detector $\mathrm{CCD}$, which constitutes the visible light receiving system. The other part is connected with a fiber optic spectrometer to form a hyperspectral receiving system. The two systems are shown in figure 4 , figure 5 and figure 6 respectively. Some optical system indicators have been listed in table 1 , which will not be detailed here. The optometric system enters the pupil at $160 \mathrm{~mm}$, and the actual optometric system corrects the chromatic aberration at $400 \mathrm{~nm}-1100 \mathrm{~nm}$ wide band. The same optical material cannot achieve high imaging quality. So the system USES two optical materials, one is fused quartz, the other is $\mathrm{CaF} 2$. Considering that $\mathrm{CaF} 2$ material is soft, relatively difficult to process and easy to deliquescence, only one $\mathrm{CaF} 2$ lens is used in the optical system, and the moisture-proof film system needs to be coated in the application to ensure the imaging quality of the optical system. The receiving system can be directly measured to the target at night. Under daytime lighting conditions, the stray light outside the band needs to be filtered out. Before the photomultiplier tube, the bandpass filter of a specific band needs to be introduced. Filter substrate material is fused quartz, In application, an uncoated fused quartz plate of equal thickness is directly used to compensate the aberration, and the filter wheel is used to realize the switching of each quartz plate.

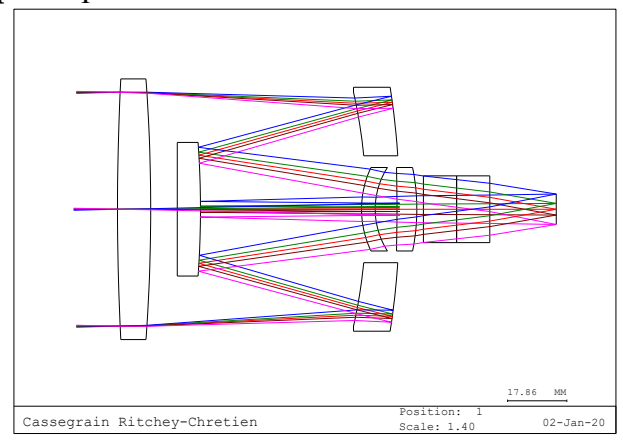

Fig. 4. visible light camera reception

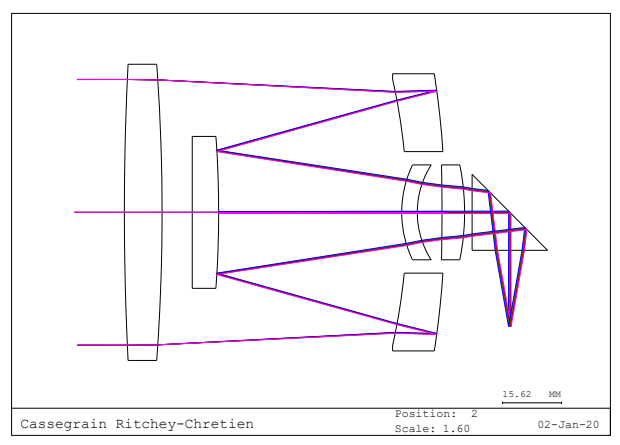

Fig. 5. hyperspectral

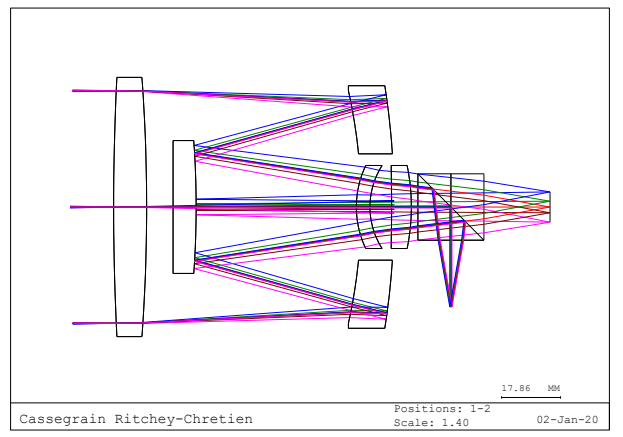

Fig. 6. combination structure of the whole machine

\subsection{Image quality evaluation of telescopic system}

The hyperspectral detector and visible light detector share a telescopic system, so only the imaging quality of the telescopic system can be analyzed. The optical path difference characteristic curve (OPD) of the telescopic system is shown in Fig. 7, where the vertical axis refers to the path difference between the ray and the main ray. Here, the path difference returned to the aperture of the system is taken as a reference, and the horizontal axis refers to the normalized entry pupil coordinates. By analyzing the data in the figure, it can be seen that for this system, the optical path difference between $400 \mathrm{~nm}$ and $1100 \mathrm{~nm}$ is less than one wavelength, which meets the requirements of imaging application. 


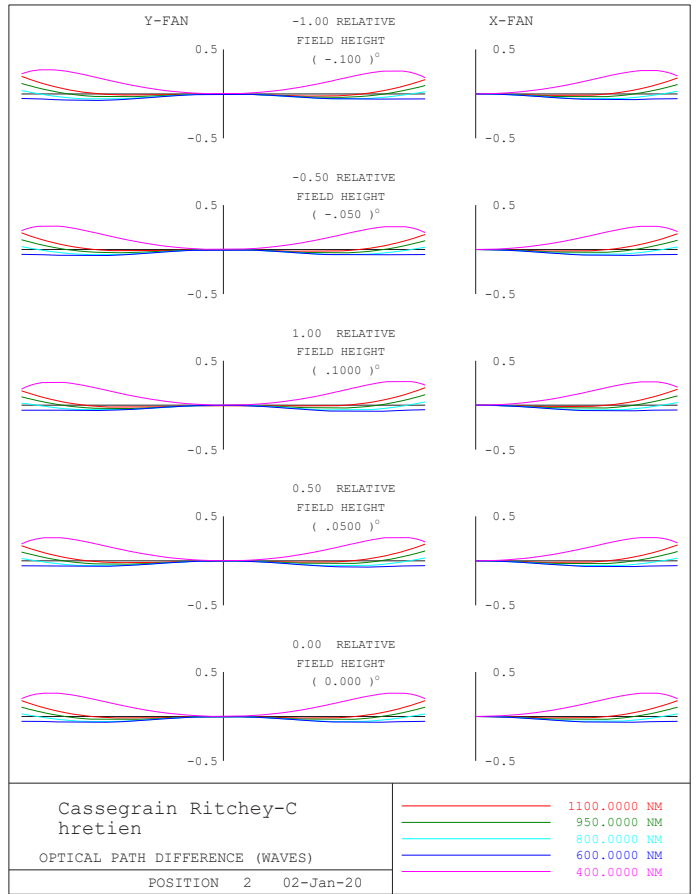

Fig. 7. characteristic curve of optical path difference of visible light receiving system

\section{SNR estimation}

\subsection{Signal-to-noise ratio of visible light camera}

Signal-to-noise ratio (SNR) is an important index to evaluate the imaging performance of lidar, which reflects the detection ability of lidar for radiation. The analysis and estimation of signal-to-noise ratio (SNR) have important referential meanings for optical system parameters, imaging device selection and subsequent electronic system design.

The process of lidar imaging of targets is actually the radiological transmission and photoelectric conversion process of laser radiation passing through atmosphere, target reflection, passing through atmosphere again and acting on the optical system before reaching the imaging detector and being received, processed and quantified. During the transmission process, lidar is not only affected by the target spectral albedo, but also affected by the absorption and scattering of laser radiation energy by various components in the atmosphere.

The signal to noise ratio of lidar is determined by the spectral response and system noise of lidar system. Considering that the detection range of the hyperspectral lidar is $0.4-0.8 \mathrm{~km}$, the thermal radiation of the target within this range can be ignored. Let the reflectance of the target be lambda $\rho(\lambda)$, and consider it as a lambert reflector. The spectral exposure of the laser radiation on the ground is $E(\lambda)$. Strictly speaking, it should be the sum of direct solar lightES $(\lambda)$ and sky scattered light $\operatorname{ED}(\lambda), \operatorname{E}(\lambda)=\operatorname{ES}(\lambda)+\operatorname{ED}(\lambda)$. Then the light spectrum brightness of the target reflected to the lidar visible light camera is:

$$
\begin{gathered}
B(\lambda)=\frac{1}{\pi} E(\lambda) \sin \theta \rho(\lambda) \tau_{s}(\lambda)+L(\lambda) \\
\omega=\alpha \beta=\frac{A}{R^{2}}=\frac{d_{1} d_{2}}{f^{\prime}} \\
\Phi(\lambda)=\pi\left(\frac{D_{0}}{2}\right)^{2} \frac{A}{R^{2}} B(\lambda)
\end{gathered}
$$

$\mathrm{D}_{0}$ is the effective optical aperture of lidar.

$$
\begin{gathered}
P(\lambda)=\frac{\pi}{4} D_{0}{ }^{2} \frac{d_{1} d_{2}}{f^{\prime 2}} \tau_{0}(\lambda) B(\lambda)=\frac{\pi}{4}\left(\frac{f^{\prime}}{F}\right)^{2} \frac{d_{1} d_{2}}{f^{\prime 2}} \tau_{0}(\lambda) B(\lambda) \\
=\pi \frac{d_{1} d_{2}}{4 F^{2}} \tau_{0}(\lambda) B(\lambda) \\
S_{e}=\frac{\pi A_{d} t_{i n t}}{4 F^{2} h c} \int_{\lambda 1}^{\lambda 2} \lambda \tau_{o} \eta_{e}(\lambda) B(\lambda) d \lambda \\
\mathrm{N}_{\mathrm{e}}=\sqrt{\mathrm{S}_{\mathrm{e}}+\sigma_{\mathrm{R}}^{2}+\mathrm{D}_{\mathrm{e}}} \\
S N R=\frac{S_{e}}{N_{e}}=\frac{S_{e}}{\sqrt{S_{e}+\sigma_{R}^{2}+D_{e}}}
\end{gathered}
$$

The oil-measuring lidar is mainly applied at night. When the operating distance is $100 \mathrm{~m}$, the corresponding target size is $100 \mathrm{~mm}$ (after the laser beam diverges).

$$
L(\lambda)=\rho \cdot E(\lambda) / \Omega
$$

The total flux received by the system can be obtained by integrating the region of the visible spectrum within the detection range. Due to the future application of lidar to the platform of small unmanned aerial vehicles (uavs), the operating range of lidar is less than $100 \mathrm{~m}$, so the atmospheric transmittance of visible light waves is not considered.According to the laser beam expanding area (100m@100m), the spectral radiation brightness at the entrance pupil of the lidar is calculated by formula (13), as shown in figure 8 .

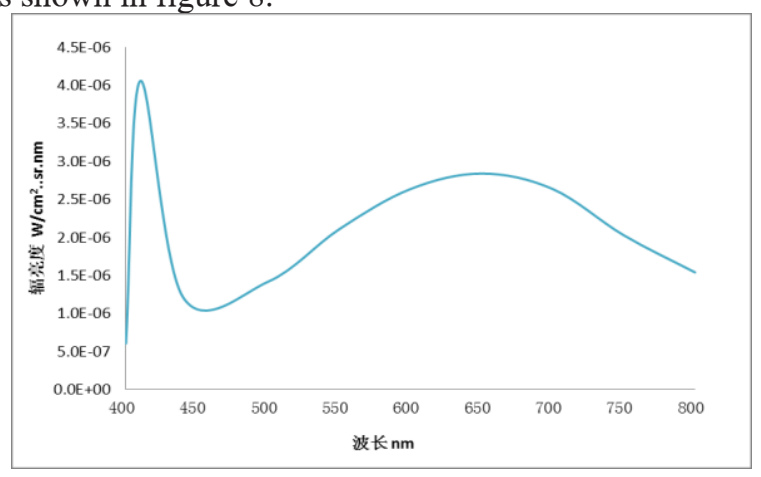

Fig. 8. The spectral radiance at the pupil of the lidar

\subsection{Signal-to-noise ratio of visible light camera}

For the hyperspectral detector, the Toshiba TCD1304AP linear array detector is adopted. There are 3684 detector pixels with an area of $8 \mathrm{~m} \times 200 \mathrm{~m}$. The spectrometer covers the band range of $200 \mathrm{~nm}-1100 \mathrm{~nm}$, and each pixel corresponds to the spectral range of $0.22 \mathrm{~nm}$. According to the formula of signal to noise ratio(12), the final signal noise of the hyperspectral detector can be obtained as shown in figure 10, and the calculated parameters are shown in table 7 . Wherein, the integrating time of the hyperspectral detector is $0.3 \mathrm{~s}$. 


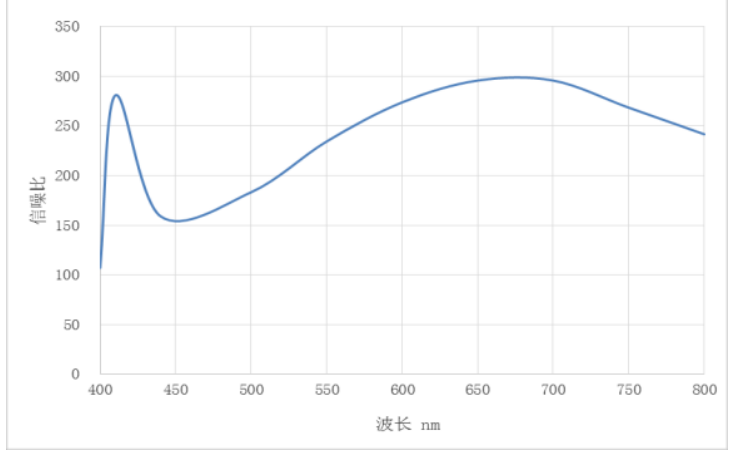

Fig. 9. signal to noise ratio of hyperspectral detector

\section{5 summary}

According to the current demand of offshore oil pollution monitoring, it is proposed to develop a supercontinuous spectrum lidar capable of obtaining both spectral information and two-dimensional images, which can cover the wave range of $400 \mathrm{~nm}-1100 \mathrm{~nm}$. Use the theory of optics and optical design software for hyperspectral excitation light radar optical design and calculation. The design completed a catadioptric optical path laser radar system, using catadioptric system structure, and greatly simplifies the structure of optical system. In order to meet the target tracking and fine structure measurement, on the basis of common optical path, to join the optical receiving system, realized the high spectrum and also had the capability of optical imaging. At the same time, the signal-to-noise ratio of the instrument is estimated, the continuous absorption, molecules, aerosol scattering, earth curvature and other factors are simulated, the influence on the calculation results is obtained, and the spectral radiation brightness at the entrance pupil of the lidar is calculated step by step.

\section{Reference}

1. Alam M S, Sidike P. Trends in oil spill detection via hyperspectral imaging[C]//2012 7th International Conference on Electrical and Computer Engineering. IEEE, 2012: 858-862.

2. Zhao sheng, Han longjiang, Ding kai, Li xiuren, Wen guoyi, Du Ming, Liang detian, Bao mengmeng, Wang taisen, Zhang jimin, Sha jingjing. Study on the impact of oil spill pollution from Qingdao donghuang oil pipeline on the environment ofJiaozhou bay [J]. Ocean and lake bog bulletin,2019(05):64-72.

3. Wei dongming. Assessment of the effectiveness of oil spill emergency materials and emergency waste disposal technology [D]. Dalian university of technology, 2014[4]

4. Chen juntian, Li shujiang, Sun junchuan, et al. Progress in numerical simulation of drift dispersion of hazardous chemicals at sea [J]. Ocean science, 2018, 42(7): 158-166
5. Yu H, Wang Q, Zhang Z, et al. The Oil spill detection using hyperspectral infrared camera [C] / / infrared and Millimeter Wave, and Terahertz Technologies IV. The International Society for called and Photonics,2017, 10030:100301G.

6. Viallefont-Robinet F, Moussous A, Déliot P, et al. Analysis of water-in-oil emulsion hyperspectral signature: contribution of pool experiment[C] //Remote Sensing of the Ocean, Sea Ice, Coastal Waters, and Large Water Regions 2018. International Society for Optics and Photonics, 2018, 10784: 107840C.

7. Ren guangbo, Guo jie, Ma yi, et al. Hyperspectral remote sensing detection and thickness estimation method of unmanned aerial vehicle (uav) for oil spill [J]. Acta oceanologica sinica, 2019, 41(5): 146-158. (in Chinese with English abstract)

8. Liu delian, Han liang, Zhang jianqi. Study on automatic detection method of oil spill in hyperspectral image [J]. Spectroscopy and spectral analysis, 2013, 33(11): 3116-3119.

9. Jiang $\mathrm{f} h$, Zhao $\mathrm{m} \mathrm{m}$, Han $\mathrm{b}$, et al. Application of synchronous fluorescence spectrometry in oil spill identification $[\mathrm{J}]$. Spectroscopy and spectral analysis, 2011, 31(1): 154-157. (in Chinese) 